\title{
Cardiovascular morbidity and mortality as a confounder of prognosis in thyroid cancer
}

\author{
Elena Izkhakov ${ }^{1,2}$, Lital Keinan-Boker ${ }^{3,4}$, Naftali Stern ${ }^{1,2,5}$ \\ 1 Institute of Endocrinology, Metabolism and Hypertension1, Tel Aviv-Sourasky Medical Center, Tel Aviv 6423906, Israel. \\ ${ }^{2}$ Sackler Faculty of Medicine, Tel Aviv University, Tel Aviv 69978, Israel. \\ ${ }^{3}$ Israel Center for Disease Control, Ministry of Health, Sheba Medical Center, Tel Hashomer Ramat - Gan 5262160, Israel. \\ ${ }^{4}$ School of Public Health, Faculty of Social Welfare and Health Sciences, University of Haifa, Haifa 3498838, Israel. \\ ${ }^{5}$ The Sagol Epigenetics Center, Tel Aviv-Sourasky Medical Center, Tel Aviv 6423906, Israel.
}

\begin{abstract}
Correspondence to: Dr. Naftali Stern, The Sagol Epigenetics Center, Institute of Endocrinology, Metabolism and Hypertension, Tel Aviv-Sourasky Medical Center and Sackler Faculty of Medicine, Tel Aviv University, 6 Weizmann street, Tel Aviv 6423906, Israel.E-mail: naftalis@tlvmc.gov.il;nafstern66@gmail.com
\end{abstract}

How to cite this article: Izkhakov E, Keinan-Boker L, Stern N. Cardiovascular morbidity and mortality as a confounder of prognosis in thyroid cancer. J Cancer Metastasis Treat 2021;7:24. https://dx.doi.org/10.20517/2394-4722.2021.24

Received: 28 Jan 2021 Accepted: 22 Apr 2021 Published: 8 May 2021

Academic Editor: Jerome M. Hershman Copy Editor: Yue-Yue Zhang Production Editor: Yue-Yue Zhang

\begin{abstract}
Thyroid cancer (TC) constitutes more than $95 \%$ of all endocrine tumors. Differentiated thyroid carcinoma (DTC), which includes the papillary and follicular types, constitutes about $90 \%$ of all TCs and $2.1 \%$ of all cancers. The incidence of DTC has increased significantly worldwide, with papillary cancer leading this trend and being the most prevalent. Whereas the prognosis of patients with DTC is generally favorable, with the overall 5-year survival rate reaching up to $95 \%$, long-term follow-up also discloses increased propensity for cardiovascular and cerebrovascular risk factors, morbidity, and mortality. Cardiovascular events are linked to TC particularly in subjects aged 45 years or more. The present review and analysis seek to highlight the significance of cardiovascular disease in the overall prognosis among TC survivors and explore potential mechanisms which might link treatment choices in DTC to cardiovascular risk and disease outcome.
\end{abstract}

Keywords: Thyroid cancer, cardiovascular morbidity, cardiovascular mortality, cardiovascular risk factors 


\section{INTRODUCTION}

Recent trends show a significant increase of the incidence of thyroid cancer $(\mathrm{TC})^{[1,2]}$. The most common type of TC is differentiated thyroid cancer (DTC), which includes follicular and papillary types and constitutes about $90 \%$ of all TC. DTC usually occurs in individuals younger than 50 years of age. Females are more affected than males, with a ratio of 3:1. DTC patients can expect a reassuring prognosis, with a 5year survival rate reaching $90 \%-95 \%{ }^{[3,4]}$. Taken together, these trends highlight the importance of studying long-term morbidity for the increasing population of DTC survivors.

The traditional treatment regimens for DTC have included thyroidectomy, radioactive iodine therapy (I131), and thyroid stimulating hormone (TSH) suppression therapy (subclinical exogenous hyperthyroid state) with levothyroxine (LT4) according to the extent of the disease ${ }^{[5]}$. Lately, there has been a gradual shift towards a more conservative, personalized approach for some low-risk subjects, ranging from watchful surveillance for isolated very small lesions (e.g., $<10 \mathrm{~mm}$ ) to lobectomy only, withholding of radioiodine treatment and/or lesser suppression of TSH (e.g., 0.5-2 $\mu \mathrm{IU} / \mathrm{mL}$ ) for low-risk subjects undergoing lobectomy only and/or no imaging or biochemical evidence for residual disease during follow-up ${ }^{[6]}$.

Pharmacological suppression of TSH proved useful in reducing recurrence and mortality in clinically detected differentiated thyroid cancer ${ }^{[]]}$. However, recent cumulative evidence supports a strong correlation between endogenous hyperthyroidism and higher mortality rates associated with cardiovascular heart disease and cerebrovascular disease in patients who have multinodular goiter and those treated with radioactive iodine, in comparison to the general population ${ }^{[8-11]}$. Furthermore, even endogenous subclinical hyperthyroidism is apparently associated with an increased risk of coronary heart disease and mortality ${ }^{[12]}$. Lastly, a low serum TSH concentration, presumably indicative of hyperthyroxinemia, was linked with a 3fold higher risk for atrial fibrillation (AF) during the following decade in subjects $\geq 60$ years of age ${ }^{[13]}$.

This review aims to provide updated data on all-cause cardiovascular mortality and cardiovascular morbidity and present pathophysiological mechanisms that are considered as probably contributing to these conditions among TC survivors.

\section{CARDIOVASCULAR AND ALL-CAUSE MORTALITY}

TSH suppression therapy is the mainstay treatment for decreasing the risk of recurrence among patients who are at high-risk of DTC. Klein Hesselink et al. ${ }^{[14]}$ evaluated the risk of cardiovascular and all-cause mortality among 524 Dutch DTC survivors compared to 1572 matched controls. A median 8.5-year followup period revealed 100 deaths among DTC survivors and 85 deaths among the controls. The authors reported a 3.3-fold increased risk of cardiovascular mortality ( $4.2 \% v$ s. $1.5 \%$, respectively) and a 4.4 -fold increased risk of all-cause mortality ( $19.1 \%$ vs. $5.4 \%$, respectively) among DTC survivors. Mortality from DTC constituted 7.4\%. In that study, each 10-fold decrease in geometric mean TSH level was independently linked to a 3.1-fold elevated cardiovascular mortality. A nomogram composed of data from the Surveillance, Epidemiology and End Results program predicted the risk of death among 29,225 TC survivors ${ }^{[15]}$. The 5year probability of mortality was $1.9 \%$ due to thyroid cancer, $0.8 \%$ due to another cancer, and $1.7 \%$ due to non-cancer causes after a median follow-up of 7 years. The most common causes of mortality, which did not result from cancer among TC survivors, were heart disease (39\%) and cerebrovascular disease (10.4\%). Data from an Israeli population-based study of 5677 TC survivors and 23,962 matched controls reveal a higher risk of all-cause mortality among the TC survivors ( $10.2 \%$ vs. $5.2 \%$, respectively, $\mathrm{HR}=1.89,95 \% \mathrm{CI}$ : 1.71-2.1; (abbreviated in all following items to the HR value followed by the 95\% CI range of values in square parenthesis $)^{[16]}$, which was associated with an elevated prevalence of cardiovascular disease $(33.6 \%$ vs. $22.3 \%, P=0.05)$, hypertension $(14.6 \%$ vs. $10.3 \%, P=0.002)$ and dyslipidemia $(11.4 \% v s .7 .2 \%, P<0.001)$ at 
the end of the surveillance period. Moreover, the number of cardiovascular risk factors correlated positively with the risk of mortality among the TC survivors, with 2-4 factors increasing the risk to a level of significance $(\mathrm{HR}=1.23[1.03-1.47], 1.66$ [1.38-1.99], and 2.59 [2.11-3.19], respectively).

Several studies on TC survivors who were receiving TSH suppression therapy have shown impairments in cardiac structure ${ }^{[17,18]}$, diastolic function ${ }^{[19-22]}$, and systolic function ${ }^{[23]}$; an increased prevalence of $\mathrm{AF}^{[24]}$; and an increased prevalence of prothrombotic condition ${ }^{[25]}$. Contrarily, Pajamäki et al ${ }^{[26]}$ found a $27 \%$ decreased cardiovascular mortality risk among 901 DTC survivors in comparison to 4485 matched controls in a Finnish population-based study during a median 18.8-year follow-up period. One of the authors' explanations for this finding was that the lifelong follow-up of DTC survivors possibly facilitated earlier determination and treatment of cardiovascular risk factors compared to the general population.

\section{Cardiovascular morbidity}

Several large retrospective studies on cardiovascular morbidity among TC survivors were published over the last few years. Pajamäki et al. ${ }^{[26]}$ found an increase in the risk of any cardiovascular disease (HR = 1.16 [1.051.28]) among 901 Finnish DTC survivors in comparison to matched controls. Suh et al. ${ }^{[27]}$ investigated the incidence of coronary heart disease among 182,419 Korean TC survivors and 182,419 non-cancer controls in a mean 4.3-year follow-up period. The TC survivors had an increased risk for coronary heart disease (HR $=1.15[1.1-1.22])$, and it was dependent upon high-dose levothyroxine $(\mathrm{HR}=1.47$ [1.34-1.6] $)$.

Izkhakov et al. ${ }^{[28]}$ assessed the atherosclerotic cardiovascular outcome among 5677 Israeli TC survivors in comparison to 23,962 adults with no thyroid disease in a mean 7.6-year follow-up period. In that study, TC survivors had an elevated risk of new atherosclerotic cardiovascular events before $(\mathrm{HR}=1.20$ [1.08-1.34]) and after $(\mathrm{HR}=1.18$ [1.05-1.33]) exclusion of patients with past cardiovascular pathology in both groups. In addition, TC survivors who developed cardiovascular events had a higher incidence of hypertension and hyperlipidemia.

Zoltek et al. ${ }^{[29]}$ compared cardiovascular incidence among 6900 DTC survivors to the general Swedish population. They revealed more hospitalizations for AF (SIR = 1.66, 95\%CI: 1.41-1.94), but there was no difference in hospitalization rates between DTC survivors and the general population with regard to other studied cardiovascular diseases.

Among people $>60$ years of age, an endogenously suppressed TSH level could lead to congestive heart failure with poor outcome $\mathrm{e}^{[30]}$. Blackburn et al. ${ }^{[31]}$ investigated the risks of aging-related diseases among 3706 TC survivors, diagnosed before and after age 40, compared to 15,587 matched cancer-free individuals from Utah Population Database. The authors found increased risk for cardiovascular morbidity in both age groups 1-5 years after cancer diagnosis compared to the matched cancer-free participants $(\mathrm{HR}=1.76[1.40-$ $2.21] v s . \mathrm{HR}=1.49$ [1.38-1.6]). Survivors $<40$ had a higher risk of hypertension $(\mathrm{HR}=2.03$ [1.75-2.32] vs. $\mathrm{HR}=1.58[1.48-1.68])$, but there was no difference in rate of congestive heart failure among both age groups of TC survivors and the matched cancer free participants.

\section{$\mathrm{AF}$}

A number of investigations found a high incidence of AF among DTC survivors. Pajamäki et al. ${ }^{[26]}$ reported an increased risk of all types of arrhythmia ( $\mathrm{HR}=1.25$ [1.06-1.48]) in association with AF $(\mathrm{HR}=1.27$ [1.061.57]) among 901 Finnish DTC survivors in comparison to matched controls during a median 18.8-year surveillance period. In a subgroup analysis, low TSH levels were linked with an increased risk of AF. Suh et al. ${ }^{[27]}$ revealed an elevated risk for AF among TC survivors compared to controls when the dosage of 
levothyroxine was high. Specifically, TC survivors who received $<115,115-144,145-169$ and $>170 \mu \mathrm{g} /$ day presented with an increased risk of AF (adjusted HR = 1.43 [1.27-1.62], 1.41 [1.26-1.58], 1.66 [1.49-1.85], and 1.78 [1.6-1.98], respectively). Zoltek et al. ${ }^{[29]}$ reported a 2 -fold higher hospitalization rate for AF among 6900 DTC survivors compared to the general Swedish population during the first 5 surveillance years, and an increased AF rate of $\sim 2-2.5$ fold among DTC patients aged 54 years or less.

\section{Cerebrovascular morbidity}

Suh et al. ${ }^{[27]}$ investigated cardiovascular morbidity as well as the incidence of ischemic stroke. TC survivors had an elevated risk of ischemic stroke ( $\mathrm{HR}=1.15$ [1.09-1.22]) which was more prominent in the group of TC survivors who were treated with levothyroxine at a dosage of $170 \mu \mathrm{g} /$ day or more $(\mathrm{HR}=1.56[1.42$ $1.72])$.

Izkhakov et al. ${ }^{[28]}$ also assessed cerebrovascular outcome among DTC survivors and found an independent risk factor of new cerebrovascular events ( $\mathrm{HR}=1.19$ [1.04-1.37]) compared to matched controls. The increased risk for new cerebrovascular event remained high $(\mathrm{HR}=1.16$ [1.01-1.36]) after those with a history of cardiovascular and cerebrovascular events were excluded.

Zoltek et al ${ }^{[29]}$ reported a higher hospitalization rate for cerebrovascular disease (SIR $=1.2,95 \%$ CI: $1.04-$ 1.38) among female DTC survivors. Toulis et al. ${ }^{[32]}$ assessed the potential cardiovascular effect of exogenous hyperthyroidism among 3009 British DTC survivors with no cardiovascular disease and 11,303 matched controls. After a median 5-year follow-up, no difference was found in the risk of ischemic heart disease, although the risk of AF and stroke was higher among the DTC survivors compared to the controls ( $\mathrm{HR}=$ 1.71 [1.36-2.15] and 1.34 [1.05-1.72], respectively). These findings warrant periodic screening for AF among TSH-suppressed DTC survivors.

\section{Associations between potential cardiovascular risk factors and cardiovascular/cerebrovascular morbidity among TC survivors}

Several pathological and high-risk conditions have been found among individuals with TC in comparison to the healthy controls. Park et al.$^{[33]}$ investigated the relationship of potential risk factors and treatment effects with cardiovascular disease outcome among 3822 TC survivors, using the state-wide Utah Population Database during a mean 8.4-year follow-up. They found a significant association between the following factors and a high risk of cardiovascular conditions: age at diagnosis (40-65 years, $\mathrm{HR}=1.66$ [1.46-1.88]; and $>65$ years, $\mathrm{HR}=2.84$ [2.46-3.27], compared to $<40$ years), male sex (compared to female sex, $\mathrm{HR}=1.46$ [1.31-1.62]), TSH suppression therapy (compared to no therapy, HR $=1.25[1.12-1.4]$ ), overweight and obesity (compared to normal BMI, HR $=1.24$ [1.11-1.39] and 1.41 [1.25-1.6], respectively), the presence of any comorbidity (compared to none, $\mathrm{HR}=4.47$ [3.87-5.15]), and $\mathrm{TC}$ distant metastasis (compared to localized TC, HR = $1.35[1.03-1.77])$.

Izkhakov et al. ${ }^{[28]}$ likewise reported an elevated risk for cardiovascular morbidity among DTC survivors aged 55-64 $(\mathrm{HR}=1.29[1.08-1.53])$ and 65-74 years $(\mathrm{HR}=1.36[1.14-1.61])$ compared to matched controls. The increased risk in those age groups persisted after individuals with past cardiovascular events were excluded $(\mathrm{HR}=1.32$ [1.09-1.60] and 1.41 [1.16-1.72], respectively). In addition, there was an association between low free thyroxin 3 (FT3) and low FT4 levels at baseline and elevated risk for new atherosclerotic cardiovascular events at the end of the study $(\mathrm{HR}=1.38[1.16-1.64]$ and $1.02[1.01-1.04]$, respectively). The FT3/FT4 ratio at baseline showed a trend of an inverse correlation with new atherosclerotic cardiovascular events $(P=$ 0.067). 


\section{Association between I-131 therapy and cardiovascular/cerebrovascular morbidity}

Lin et al ${ }^{[34]}$ retrospectively evaluated the association between I-131 therapy for TC and stroke risk in 10,104 Taiwanese DTC survivors (mean age 49.4 years). The participants were classified into two groups that did and did not receive I-131 therapy. The mean stroke latency after I-131 therapy was 6.6 years compared to 6.59 years without I-131 therapy. The risk of ischemic or hemorrhagic stroke was not greater in the group who received I-131 therapy after a 10-year follow-up.

Kim et al ${ }^{[35]}$ recently assessed the adverse effects of I-131 therapy on cardiovascular and cerebrovascular diseases among 4845 TC patients aged 40-79 years. Overall, 2533 DTC patients (52.3\%) received I-131 therapy and the median I-131 cumulative dosage was $103 \mathrm{mCi}$. After a median 5.5-year follow-up, no significant difference was found in the risk of ischemic or hemorrhagic stroke, ischemic heart disease, or heart failure among DTC survivors who received I-131 therapy and those who did not. In addition, the I131 accumulative dosage was not associated with cardiovascular morbidity among the TC survivors.

\section{Metabolic, resting energy expenditure, and body composition changes}

The major role of thyroid hormones in the regulation of rest energy expenditure (REE), body composition, and body weight has been well documented ${ }^{[36]}$. Several studies have found a positive association between TSH and FT3 and metabolic parameters, obesity, and body mass index (BMI) in euthyroid adults ${ }^{[37,38]}$.

Izkhakov et al. ${ }^{[39]}$ assessed body composition, body weight, metabolic parameters, respiratory quotient (RQ), and REE changes among 15 female DTC patients starting from the endogenous euthyroid state prior to total thyroidectomy and continuing throughout the following year of an exogenous subclinical hyperthyroid state. The major findings of that study were that the standard protocol of thyroidectomy and I-131 therapy followed by TSH suppression with LT4 resulted in elevation of REE, while there were no meaningful changes in BMI and lean body mass (LBM). Additionally, the following significant changes were found: increases in heart rate, systolic and diastolic blood pressures, and FT4 levels, while RQs, TSH levels, and $\mathrm{TT} 3 / \mathrm{FT} 4$ ratios decreased in comparison to the endogenous euthyroid state at the beginning of the followup. There was a subsequent negative relationship between the TT3/FT4 ratio and BMI, fasting blood glucose, systolic blood pressure, android fat distribution, REE, and REE/LBM, while a positive relationship between $\mathrm{TT} 3 / \mathrm{FT} 4$ ratio and RQ was revealed.

An earlier investigation by Wolf et al. ${ }^{[40]}$ included 29 female and 12 male DTC survivors after thyroidectomy and TSH suppressive LT4 therapy, and there were non-significant changes in REE and in body composition in the DTC group compared to the matched control group.

\section{CONCLUSION}

Despite the excellent overall prognosis in TC, afflicted patients are at higher risk for unfavorable metabolic changes, cardiovascular and cerebrovascular morbidity, and long-term cardiovascular and all-cause mortality. Furthermore, this appears to be at least partially related to the unique state of post-thyroidectomy and intentionally induced hyperthyroidism, with attempted suppression of circulating TSH. Collectively, the data reviewed herein suggest that benefits in terms of TC treatment outcome should be weighed against significant treatment-related cardiovascular risk. This highlights the need for personally tailored decisions regarding both surgical and medical therapeutic strategies in TC.

The first question that arises regarding the therapeutic management of patients with TC concerns the extent of thyroid removal, hemithyroidectomy, or total thyroidectomy. The choice of operation can radically affect the patient's future comorbidities. According to recently published American guidelines, patients at low risk 
of recurrence may be treated by hemithyroidectomy, with laboratory and neck ultrasound follow-up ${ }^{[5]}$. That approach allows production of $\mathrm{T} 4$ and $\mathrm{T} 33$ by the residual thyroid tissue and reduces the negative adverse effects of replacement therapy, which depends solely upon LT4 treatment. A study by Ito et al. ${ }^{[41]}$ showed a positive effect of the maintenance of the postoperative serum T3 level in LT4-treated patients after hemithyroidectomy compared to those who underwent total thyroidectomy. In a descriptive study of 15 women with newly diagnosed DTC, negative changes in metabolic factors, REE, and body composition were observed under standard care during the first year following the diagnosis ${ }^{[39]}$. Those changes paralleled decreases in TSH and the TT3/FT4 ratio, implying a higher prevalence of cardiovascular risk factors ${ }^{[39]}$. In healthy adults, exogenous increased TT3 was found to decrease fat and fat-free mass, elevate the energy expenditure ${ }^{[42]}$, and elevate thermogenesis in skeletal muscle by promoting the uncoupling of mitochondrial energy $^{[43]}$.

A decision to perform total thyroidectomy necessarily entails three critical treatment dilemmas: (1) the need for radioactive iodine treatment; (2) the type of hormone replacement therapy; and (3) the need for TSH suppression, as well as its extent and duration. The current standard hormone replacement therapy is with LT4. Based on the results of previous investigations ${ }^{[39,42,43]}$, we suggest considering the addition of T3 for cases in which there is a significant decline in the $\mathrm{T} 3 / \mathrm{T} 4$ ratio.

Endogenously suppressed TSH was found to be associated with cardiovascular morbidity and mortality in the general population ${ }^{[8,9]}$. The findings of elevated atherosclerotic cardiovascular risk factors as well as cardiovascular morbidity among TC patients also highlight the importance of a balance between benefits and detriments when considering exogenous TSH suppression for TC patients ${ }^{[26,27,33]}$. The extent of TSH suppression has also been questioned by other ${ }^{[44]}$. Recently issued research and guidelines advocate such treatment only for patients at high risk of DTC recurrence ${ }^{[5]}$. The clinical implication of these findings is that TC survivors are at higher risk for cardiovascular conditions. As such, cardiovascular risk factors need to be scrupulously monitored among TC survivors.

\section{DECLARATIONS}

\section{Authors' contributions}

Conceived and wrote the article: Izkhakov $\mathrm{E}$

Edited the article: Keinan-Boker L

Conceived and edited the article: Stern N

\section{Availability of data and materials}

Not applicable.

\section{Financial support and sponsorship}

None.

\section{Conflicts of interest}

All authors declared that there are no conflicts of interest.

\section{Ethical approval and consent to participate}

Not applicable.

\section{Consent for publication}

Not applicable. 


\section{Copyright}

(c) The Author(s) 2021.

\section{REFERENCES}

1. Davies L, Welch HG. Current thyroid cancer trends in the United States. JAMA Otolaryngol Head Neck Surg 2014;140:317-22. DOI PubMed

2. La Vecchia C, Malvezzi M, Bosetti C, et al. Thyroid cancer mortality and incidence: a global overview. Int J Cancer 2015;136:218795. DOI PubMed

3. Konturek A, Barczyński M, Stopa M, Nowak W. Trends in prevalence of thyroid cancer over three decades: a retrospective cohort study of 17,526 surgical patients. World J Surg 2016;40:538-44. DOI PubMed PMC

4. Kitahara CM, Sosa JA. The changing incidence of thyroid cancer. Nat Rev Endocrinol 2016;12:646-53. DOI PubMed

5. Haugen BR, Alexander EK, Bible KC, et al. 2015 American thyroid association management guidelines for adult patients with thyroid nodules and differentiated thyroid cancer: the American thyroid association guidelines task force on thyroid nodules and differentiated thyroid cancer. Thyroid 2016;26:1-133. DOI PubMed PMC

6. Filetti S, Durante C, Hartl D, et al. Thyroid cancer: ESMO clinical practice guidelines for diagnosis, treatment and follow-up. Ann Oncol 2019;30:1856-83. DOI PubMed

7. Mazzaferri EL, Young RL. Papillary thyroid carcinoma: a 10 year follow-up report of the impact of therapy in 576 patients. Am J Med 1981;70:511-8. DOI PubMed

8. Hall P, Lundell G, Holm LE. Mortality in patients treated for hyperthyroidism with iodine-131. Acta Endocrinol (Copenh) 1993;128:230-4. DOI PubMed

9. Franklyn JA, Maisonneuve P, Sheppard MC, Betteridge J, Boyle P. Mortality after the treatment of hyperthyroidism with radioactive iodine. N Engl J Med 1998;338:712-8. DOI PubMed

10. Franklyn JA, Sheppard MC, Maisonneuve P. Thyroid function and mortality in patients treated for hyperthyroidism. JAMA 2005;294:71-80. DOI PubMed

11. Metso S, Jaatinen P, Huhtala H, Auvinen A, Oksala H, Salmi J. Increased cardiovascular and cancer mortality after radioiodine treatment for hyperthyroidism. J Clin Endocrinol Metab 2007;92:2190-6. DOI PubMed

12. Collet TH, Gussekloo J, Bauer DC, et al. Subclinical hyperthyroidism and the risk of coronary heart disease and mortality. Arch Intern Med 2012;172:799-809. DOI PubMed PMC

13. Sawin CT, Geller A, Wolf PA, et al. Low serum thyrotropin concentrations as a risk factor for atrial fibrillation in older persons. $N$ Engl J Med 1994;331:1249-52. DOI PubMed

14. Klein Hesselink EN, Klein Hesselink MS, de Bock GH, et al. Long-term cardiovascular mortality in patients with differentiated thyroid carcinoma: an observational study. J Clin Oncol 2013;31:4046-53. DOI PubMed

15. Yang L, Shen W, Sakamoto N. Population-based study evaluating and predicting the probability of death resulting from thyroid cancer and other causes among patients with thyroid cancer. J Clin Oncol 2013;31:468-74. DOI PubMed

16. Izkhakov E, Keinan-Boker L, Barchana M, et al. Long-term all-cause mortality and its association with cardiovascular risk factors in thyroid cancer survivors: an Israeli population-based study. BMC Cancer 2020;20:892. DOI PubMed PMC

17. Mercuro G, Panzuto MG, Bina A, et al. Cardiac function, physical exercise capacity, and quality of life during long-term thyrotropinsuppressive therapy with levothyroxine: effect of individual dose tailoring. J Clin Endocrinol Metab 2000;85:159-64. DOI PubMed

18. Shargorodsky M, Serov S, Gavish D, Leibovitz E, Harpaz D, Zimlichman R. Long-term thyrotropin-suppressive therapy with levothyroxine impairs small and large artery elasticity and increases left ventricular mass in patients with thyroid carcinoma. Thyroid 2006;16:381-6. DOI PubMed

19. Biondi B, Fazio S, Carella C, et al. Cardiac effects of long term thyrotropin-suppressive therapy with levothyroxine. J Clin Endocrinol Metab 1993;77:334-8. DOI PubMed

20. Fazio S, Biondi B, Carella C, et al. Diastolic dysfunction in patients on thyroid-stimulating hormone suppressive therapy with levothyroxine: beneficial effect of beta-blockade. J Clin Endocrinol Metab 1995;80:2222-6. DOI PubMed

21. Taillard V, Sardinoux M, Oudot C, et al. Early detection of isolated left ventricular diastolic dysfunction in high-risk differentiated thyroid carcinoma patients on TSH-suppressive therapy. Clin Endocrinol (Oxf) 2011;75:709-14. DOI PubMed

22. Gazdag A, Nagy EV, Erdei A, et al. Aortic stiffness and left ventricular function in patients with differentiated thyroid cancer. $J$ Endocrinol Invest 2015;38:133-42. DOI PubMed

23. Abdulrahman RM, Delgado V, Hoftijzer HC, et al. Both exogenous subclinical hyperthyroidism and short-term overt hypothyroidism affect myocardial strain in patients with differentiated thyroid carcinoma. Thyroid 2011;21:471-6. DOI PubMed

24. Abonowara A, Quraishi A, Sapp JL, et al. Prevalence of atrial fibrillation in patients taking TSH suppression therapy for management of thyroid cancer. Clin Invest Med 2012;35:E152-6. DOI PubMed

25. Horne MK 3rd, Singh KK, Rosenfeld KG, et al. Is thyroid hormone suppression therapy prothrombotic? J Clin Endocrinol Metab 2004;89:4469-73. DOI PubMed

26. Pajamäki N, Metso S, Hakala T, et al. Long-term cardiovascular morbidity and mortality in patients treated for differentiated thyroid cancer. Clin Endocrinol (Oxf) 2018;88:303-10. DOI PubMed

27. Suh B, Shin DW, Park Y, et al. Increased cardiovascular risk in thyroid cancer patients taking levothyroxine: a nationwide cohort study in Korea. Eur J Endocrinol 2019;180:11-20. DOI PubMed

28. Izkhakov E, Meyerovitch J, Barchana M, Shacham Y, Stern N, Keinan-Boker L. Long-term cardiovascular and cerebrovascular 
morbidity in Israeli thyroid cancer survivors. Endocr Connect 2019;8:398-406. DOI PubMed PMC

29. Zoltek M, Andersson TM, Hedman C, Ihre-Lundgren C, Nordenvall C. Cardiovascular Incidence in 6900 Patients with Differentiated Thyroid Cancer: a Swedish Nationwide Study. World J Surg 2020;44:436-41. DOI PubMed

30. Klein I, Danzi S. Thyroid disease and the heart. Curr Probl Cardiol 2016;41:65-92. DOI PubMed

31. Blackburn BE, Ganz PA, Rowe K, et al. Aging-related disease risks among young thyroid cancer survivors. Cancer Epidemiol Biomarkers Prev 2017;26:1695-704. DOI PubMed PMC

32. Toulis KA, Viola D, Gkoutos G, Keerthy D, Boelaert K, Nirantharakumar K. Risk of incident circulatory disease in patients treated for differentiated thyroid carcinoma with no history of cardiovascular disease. Clin Endocrinol (Oxf) 2019;91:323-30. DOI PubMed

33. Park J, Blackburn BE, Ganz PA, et al. Risk factors for cardiovascular disease among thyroid cancer survivors: findings from the Utah cancer survivors study. J Clin Endocrinol Metab 2018;103:2468-77. DOI PubMed PMC

34. Lin CY, Lin CL, Lo YC, Kao CH. Association between radioiodine treatment for thyroid cancer and risk of stroke. Head Neck 2017;39:2311-8. DOI PubMed

35. Kim KJ, Song JE, Kim JY, et al. Effects of radioactive iodine treatment on cardiovascular disease in thyroid cancer patients: a nationwide cohort study. Ann Transl Med 2020;8:1235. DOI PubMed PMC

36. Kim B. Thyroid hormone as a determinant of energy expenditure and the basal metabolic rate. Thyroid 2008;18:141-4. DOI PubMed

37. Iacobellis G, Ribaudo MC, Zappaterreno A, Iannucci CV, Leonetti F. Relationship of thyroid function with body mass index, leptin, insulin sensitivity and adiponectin in euthyroid obese women. Clin Endocrinol (Oxf) 2005;62:487-91. DOI PubMed

38. Roos A, Bakker SJ, Links TP, Gans RO, Wolffenbuttel BH. Thyroid function is associated with components of the metabolic syndrome in euthyroid subjects. J Clin Endocrinol Metab 2007;92:491-6. DOI PubMed

39. Izkhakov E, Vaisman N, Barnes S, Barchana M, Stern N, Keinan-Boker L. Body composition, resting energy expenditure, and metabolic changes in women diagnosed with differentiated thyroid carcinoma. Thyroid 2019;29:1044-51. DOI PubMed PMC

40. Wolf M, Weigert A, Kreymann G. Body composition and energy expenditure in thyroidectomized patients during short-term hypothyroidism and thyrotropin-suppressive thyroxine therapy. Eur J Endocrinol 1996;134:168-73. DOI PubMed

41. Ito M, Miyauchi A, Kang S, et al. Effect of the presence of remnant thyroid tissue on the serum thyroid hormone balance in thyroidectomized patients. Eur J Endocrinol 2015;173:333-40. DOI PubMed

42. Lovejoy JC, Smith SR, Bray GA, et al. A paradigm of experimentally induced mild hyperthyroidism: effects on nitrogen balance, body composition, and energy expenditure in healthy young men. J Clin Endocrinol Metab 1997;82:765-70. DOI PubMed

43. Lebon V, Dufour S, Petersen KF, et al. Effect of triiodothyronine on mitochondrial energy coupling in human skeletal muscle. $J$ Clin Invest 2001;108:733-7. DOI PubMed PMC

44. Sugitani I, Fujimoto Y. Does postoperative thyrotropin suppression therapy truly decrease recurrence in papillary thyroid carcinoma? $J$ Clin Endocrinol Metab 2010;95:4576-83. DOI PubMed 